\title{
Translocation of Lysosomal Cathepsin D Caused by Oxidative Stress or Proteasome Inhibition in Primary Cultured Neurons and Astrocytes
}

\author{
Yuri Miura, ${ }^{*, a}$ Yoko Sakurai, ${ }^{a}$ Masato Hayakawa,${ }^{a, b}$ Yukiko Shimada, ${ }^{a, 1)}$ Hans Zempel, ${ }^{a}$ Yuji Sato, ${ }^{a}$ \\ Shin-ichi HisAnaga, ${ }^{b}$ and Tamao ENDO ${ }^{a}$ \\ ${ }^{a}$ Research Team for Functional Genomics, Tokyo Metropolitan Institute of Gerontology; 35-2 Sakaecho, Itabashi-ku, \\ Tokyo 173-0015, Japan: and ${ }^{b}$ Department of Biological Sciences, Tokyo Metropolitan University; Hachioji, Tokyo \\ 192-0397, Japan. Received July 27, 2009; accepted October 5, 2009; published online October 6, 2009
}

We reported previously that $\mathrm{N}$-linked glycoproteins were accumulated in the cytosol of the normal aging rat brain, and that one protein had been identified as cathepsin D (Mech. Ageing Dev., 127, $771-778$ (2006)). In this study, to elucidate the mechanism of cathepsin D accumulation in the cytosol, we examined the effects of oxidative stress and proteasome inhibition on the apoptosis and subcellular localization of cathepsin $D$ in primary cultured neurons and astrocytes. Using 4'-6-diamidino-2-phenylindole (DAPI)- or Hoechst 33342-staining and annexin $\mathrm{V}$ detection, we found that oxidative stress caused by tert-butyl hydroperoxide and proteasome inhibition by lactacystin induced apoptosis in neurons and astrocytes. Furthermore, after cell fractionation, it was demonstrated that cathepsin D was translocated from lysosomes to cytosol under apoptosis-inducing conditions in both cells. These results suggested that oxidative stress and the suppression of proteasome activity triggered the translocation of cathepsin D from lysosomes to cytosol. The possible mechanism of age-related accumulation of cathepsin $D$ in the cytosol of the normal rat brain will be discussed.

Key words cathepsin D; oxidative stress; proteasome inhibition; N-linked glycoprotein

Proteins produced by eukaryotic cells frequently undergo various post-translational modifications, such as phosphorylation, acetylation, ubiquitination, and glycosylation. The modified proteins perform various functions involved in signal transduction, localization, and degradation signals. Among them, glycosylation is the most common protein modification in mammals. ${ }^{2)}$ In the biosynthesis of N-linked glycoprotein, sugar chains are added to nascent polypeptides and processed by various enzymes in the endoplasmic reticulum and Golgi apparatus, and the complete glycoproteins are transported to their target organelles or secreted extracellularly by means of vesicular traffic. Therefore, with some exceptions, ${ }^{3)} \mathrm{N}$-linked glycoproteins can not be found in the cytosol $^{4-6)}$; however, we have reported the accumulation of N-glycoproteins in the cytosol in both Alzheimer's disease ${ }^{7)}$ and normal aging of the rat brain. ${ }^{8}$ In a normally aging rat brain, accumulated $\mathrm{N}$-glycoproteins were detected by two-dimensional polyacrylamide gel electrophoresis and lectin blot, and one protein was identified as cathepsin $\mathrm{D}$, a lysosomal protease. ${ }^{8)}$ Cathepsin D is a major intracellular aspartic protease. In the rat brain, cathepsin D was detected in both neurons and glial cells. Increased cathepsin D has been reported in aging, ${ }^{9-11)}$ Alzheimer's disease, ${ }^{12,13)}$ and dystrophy ${ }^{14)}$; however, the physiological and pathological significance is poorly understood. In addition, the mechanism of cathepsin $\mathrm{D}$ accumulation in cytosol in the aging process remains elusive. ${ }^{8)}$

Reactive oxygen species (ROS) and reactive nitrogen species (RNS) are constantly produced in various cells and tissues. The concentrations of these reactive species are determined by redox balance between the rates of production from endogenous and exogenous origins and the rates of clearance by various antioxidant systems; however, the redox balance gradually degenerates with aging, finally resulting in oxidative stress, ${ }^{15,16)}$ which is thought to cause the dysfunction of various subcellular components, including lysosomes.
This suggests that oxidative stress may induce changes in the intracellular distribution of cathepsin D.

The accumulation of cathepsin D in the cytosol may be also induced by the dysfunction of protein degradation systems. Protein degradation systems are categorized into lysosomal and non-lysosomal systems, such as proteasomes. Proteasome protein degradation is known to eliminate misfolded/unassembled (glyco)proteins in the cytosol; therefore, the decline of proteasome activity may lead to the accumulation of cathepsin D in the cytosol.

It is important to elucidate the mechanism of cathepsin D accumulation in the cytosol of the rat brain during aging; however, it is difficult to examine the whole brain because it is too complicated. Therefore, we used primary cultured neurons and astrocytes derived from the brain, and examined whether oxidative stress and proteasome inhibition induced the accumulation of cathepsin D in the cytosol and also induced apoptosis in vitro.

\section{MATERIALS AND METHODS}

Materials Anti-cathepsin D polyclonal (R-20) and anti$\beta$ III-tubulin (TuJ-1) monoclonal antibodies were purchased from Santa Cruz Biotechnology (Santa Cruz, CA, U.S.A.) and Promega Co., (Madison, WI, U.S.A.), respectively. Antiglial fibrillary acidic protein (GFAP) monoclonal and antiglyceraldehyde-3-phosphate dehydrogenase (GAPDH) monoclonal antibodies were obtained from Chemicon International Inc. (Temecula, CA, U.S.A.). Peroxidase-conjugated and Alexa488 or 594-conjugated secondary antibodies were purchased from Santa Cruz Biotechnology and Invitrogen (Molecular Probe, Carlsbad, CA, U.S.A.), respectively. Rhodamine-conjugated secondary antibody was purchased from ICN Pharmaceutical Inc. (Cappel, Aurora, OH, U.S.A.). All other reagents were of the highest quality available.

Animals Wistar rats were obtained from SLC Japan 
(Shizuoka, Japan). All experimental procedures using animals were approved by the Animal Care and Use Committees of Tokyo Metropolitan Institute of Gerontology.

Primary Neuron Cultures Primary cultures of cortical neurons were prepared from Wistar rat embryos of 15-d gestation according to the conventional method. Briefly, after dissection, the cerebral cortex was minced and treated with dispase (Roche Diagnostics, Mannheim, Germany) for $5 \mathrm{~min}$ and DNase (Worthington Biochemical, Lakewood, NJ, U.S.A.) for $10 \mathrm{~min}$. After centrifugation, cells were resuspended in a neurobasal medium (Invitrogen) containing 50\% Dulbecco's modified Eagle medium (DMEM) (Invitrogen), $0.75 \%$ B27 supplement (Invitrogen), $50 \mathrm{~mm}$ L-glutamine, $2.5 \mathrm{~mm}$ glutamin acid, $0.5 \%$ antibiotic-antimycotic (Invitrogen), and 5\% fetal bovine serum (FBS) (HyClone; Thermo Fisher Scientific, South Logan, Utah, U.S.A.) and were subsequently dissociated by repeated pipetting through a Pasteur pipette. The cells were then plated in a poly-L-lysine-coated $10 \mathrm{~cm}$ dish at $37^{\circ} \mathrm{C}$ under a humidified atmosphere containing $5 \% \mathrm{CO}_{2} / 95 \%$ air. Culture medium was exchanged for neurobasal medium containing $1.5 \% \quad$ B27 supplement, $100 \mathrm{~mm}$ L-glutamine, $5 \mathrm{~mm}$ glutamic acid and 1\% antibioticantimycotic at days in vitro 2 (DIV 2), and further exchanged for neurobasal medium containing 1.5\% B27 supplement, $0.5 \mathrm{~mm}$ L-glutamine, and $0.5 \%$ antibiotic-antimycotic on DIV 6. Almost cells on DIV 10 were immunopositive against TuJ1 , a neuron marker.

Primary Astrocyte Cultures Astrocyte primary cultures of cerebral cortex were prepared from Wistar rat embryos of 16-d gestation. As described previously, ${ }^{17)}$ cells were dissociated with dispase I and DNAase. Dispersed cells were resuspended in DMEM containing $10 \% \mathrm{FBS}$ and $50 \mathrm{U} / \mathrm{ml}$ penicillin-50 $\mu \mathrm{g} / \mathrm{ml}$ streptomycin (Invitrogen). After being grown to confluence, the cells were trypsinized, collected, resuspended and plated in $10 \mathrm{~cm}$ dishes. The cells were $>95 \%$ immunopositive against GFAP, an astrocyte marker.

Oxidative Stress or Proteasome Inhibition In oxidative stress experiments, culture medium was exchanged for medium containing B27 supplement without antioxidants (AO (-) medium) on DIV 9, and each concentration of tertbutyl hydroperoxide (tBHP, Wako Pure Chemicals, Osaka, Japan) was added to cells for $1 \mathrm{~h}$ on DIV 10. After washing with phosphate buffered saline (PBS), cells were incubated in $\mathrm{AO}(-)$ medium for a further $24 \mathrm{~h}$ and harvested for each assay on DIV 11. In proteasome inhibition experiments, lactacystin (Calbiochem, Darmstadt, Germany) was added to cells in medium containing B27 supplement with antioxidants $(\mathrm{AO}(+)$ medium) on DIV 10 and incubated for a further $24 \mathrm{~h}$. In astrocytes, cultured medium was exchanged for serum-free medium (DMEM (-)) $24 \mathrm{~h}$ before exposure to oxidative stress, and each concentration of tBHP was loaded for $1 \mathrm{~h}$. After washing with PBS, cells were incubated in $\operatorname{DMEM}(-)$ for a further $24 \mathrm{~h}$ and harvested for each assay similarly to neurons. Lactacystin was added to astrocytes for $24 \mathrm{~h}$ in complete medium (DMEM $(+)$ ). Control cells of tBHP and lactacystin exposure were incubated in the same culture medium as tBHP-exposed cells (AO $(-)$ for neurons or DMEM ( - ) for astrocytes) and as lactacystin-loaded cells $(\mathrm{AO}(+)$ for neurons or DMEM $(+)$ for astrocytes), respectively. Control cells for tBHP and lactacystin are represented by control ( - ) and control $(+)$, respectively.

Immunocytochemical and Immunofluorescent Stainings Cultured neurons and astrocytes exposed to stress were incubated with 4'-6-diamidino-2-phenylindole (DAPI) (2 $\mu \mathrm{g} / \mathrm{ml}$, Roche Diagnostics) and Hoechst $33342(200 \mu \mathrm{M}$, Molecular Probe) for $15 \mathrm{~min}$ at $37^{\circ} \mathrm{C}$, respectively, and then fixed in methanol for $15 \mathrm{~min}$ at $-20^{\circ} \mathrm{C}$. For dual immunocytochemical staining, cells were incubated in a blocking solution consist of $0.5 \%$ BSA in PBS for $1 \mathrm{~h}$, followed by incubation with a primary antibody (Cathepsin D, 1:1000) for $1 \mathrm{~h}$ at room temperature. After washing with PBS, the cells were incubated with a secondary antibody labeled by Alexa Fluor488 $(1: 2000)$ for $1 \mathrm{~h}$ at room temperature. Next, the cells were similarly incubated with primary antibody (TuJ-1, 1:10000 for neurons or GFAP, 1:200 for astrocytes) followed by a secondary antibody labeled with Alexa Fluor594 (1:5000) for neurons or rhodamine $(1: 3000)$ for astrocytes. Finally, the coverslips were mounted on glass slides, and then visualized and photographed using a confocal laser scanning fluorescence microscope (LSM5 Pascal; Carl Zeiss, Jena, Germany) or an inverted fluorescent microscope equipped with a CCD digital camera (IX70; Olympus, Tokyo, Japan).

Flow Cytometry Apoptotic cells were detected by flow cytometry after staining with both fluorescein isothiocyanate (FITC)-conjugated annexin $\mathrm{V}$ and propidium iodide (PI), with a commercially available kit (Annexin V-FITC Apoptosis Detection Kit; BioVision Research Product, Mountain View, CA, U.S.A.). To detect both early and late apoptosis, both adherent and floating cells were harvested together. After collecting culture medium containing floating cells, adherent cells were treated with $0.05 \%$ trypsin-ethylenediaminetetraacetic acid (EDTA) for $2-10 \mathrm{~min}$ at $37^{\circ} \mathrm{C}$. The detached cells were combined with the collected floating cells. The cells were then washed by centrifugation with $2 \%$ FBS-PBS and incubated in Annexin V-FITC and PI labeling solution on ice in the dark for 5 min. Flow cytometry analysis was performed with an EPICS Altra flow cytometer equipped with a 488-nm argon laser and a 635-nm red diode laser (Coulter Electronics, Miami, FL, U.S.A.).

Subcellular Fractionation Cells incubated in $10 \mathrm{~cm}$ dishes were washed twice with PBS and harvested by a cell scraper after the addition of SET buffer $(10 \mathrm{~mm}$ Tris- $\mathrm{HCl} \mathrm{pH}$ 7.4, $1 \mathrm{~mm}$ EDTA-2Na and $250 \mathrm{~mm}$ sucrose). They were homogenized with 20 strokes of a pestle. Unbroken cells and nuclei were precipitated by centrifugation at $600 \times \boldsymbol{g}$ for $10 \mathrm{~min}$ at $4{ }^{\circ} \mathrm{C}$. After discarding the pellet, the supernatant was centrifuged at $100000 \times \mathbf{g}$ for $1 \mathrm{~h}$ at $4{ }^{\circ} \mathrm{C}$ to pellet the microsomal fraction, and the resulting supernatants were stored as cytosolic fractions. Proteins in the cytosolic fraction were concentrated by acetone precipitation. Both fractions were suspended in sample buffer consisting of $50 \mathrm{~mm}$ Tris- $\mathrm{HCl}$ (pH 6.8), 2\% SDS, 10\% glycerol, 1\% 2-ME, and 0.01\% BPB followed by boiling for $10 \mathrm{~min}$. The protein concentration was determined using the Protein Assay (Bio-Rad Laboratories, Hercules, CA, U.S.A.).

Western Blot Analysis Western blot analyses were performed as described previously. ${ }^{18)}$ The nitrocellulose membranes (Bio-Rad Laboratories) transferring the cellular proteins were incubated with primary antibodies against cathep$\sin \mathrm{D}(1: 200)$ overnight at $4^{\circ} \mathrm{C}$, or GAPDH $(1: 2000)$ for $1 \mathrm{~h}$ 
at room temperature, and with peroxidase-conjugated secondary antibodies (anti-goat IgG $1: 5000$, or anti-mouse IgG $1: 5000$ ) for $2 \mathrm{~h}$ at room temperature. The immunoreactive proteins were visualized using an ECL plus Western blotting detection system (GE Healthcare, Buckinghamshire, England) according to the manufacturer's instructions.

Statistical Analysis Experimental data are expressed as the mean \pm S.D. To compare the two groups, the unpaired $t$ test was performed. A $p$-value less than 5\% was considered significant. Microsoft Excel was used for statistical analysis.

\section{RESULTS}

Effects of Oxidative Stress and Proteasome Inhibition on Neurons First, we performed DAPI staining of chromatin and immunostaining of cathepsin D and TuJ-1 in primary cultured neurons as shown in Fig. 1a. When cells were treated with $3 \mu \mathrm{M}$ tBHP for $1 \mathrm{~h}$, chromatin condensation was observed on the DAPI staining panel, indicating apoptotic cells. Furthermore, translocation of cathepsin D from lysosome-like granules was observed in tBHP-added cells as compared to the control ( - ). The addition of $1 \mu \mathrm{M}$ lactacystin, a selective inhibitor of $20 \mathrm{~S}$ proteasome, also caused chromatin condensation and translocation of cathepsin D. Based on the previous reports, ${ }^{19-22)}$ we consider that cathepsin D was translocated from lysosome to cytosol by oxidative stress or proteasome inhibition. However, since the translocated cathepsin D was not distributed throughout the cytosol but heterogeneously distributed in the cytosol, it was likely to be included in some aggregated form. Further study relating to the distribution of cathepsin D in the cytosol is necessary in the future. The dose of tBHP or lactacystin, and the timing of cell fixation after stresses were examined under various conditions, and the optimized conditions to observe apoptosis and cathepsin D translocation were determined.

As shown in Fig. 1 (b, open bars), the percentage of apoptotic cells was increased depending on the concentration of tBHP or lactacystin. The percentage of cathepsin D translocated to the cytosol was increased together with apoptotic cells (Fig. 1 (b, closed bars)). These results demonstrated that cathepsin D was accumulated in the cytosol concomitant with apoptosis induced by oxidative stress or proteasome inhibition in primary cultured neurons.

To confirm the induction of apoptosis by oxidative stress and proteasome inhibition, we performed another set of experiments to detect phosphatidylserine on the outer membrane. As shown in Fig. 1c, apoptotic cells were analyzed by annexin V-PI staining using a flow cytometer. In the quadrant designated lower left (B3) is a group of annexin V- and PInegative cells, indicating living cells; upper left (B1) is PIpositive, indicating a group of necrotic cells; lower right (B4) is annexin $\mathrm{V}$-positive, indicating a group of early apoptotic cells; upper right (B2) is both annexin V- and PI-positive, indicating a group of late apoptotic cells. The addition of either tBHP or lactacystin decreased the percentage of B3 cells and increased that of B4 cells, indicating that oxidative stress and proteasome inhibition caused apoptosis. The rates of living cells (B3) in the control $(-)$ and control $(+)$, about $80 \%$, are lower than the control cells shown in Fig. 1b. The probable reason is that the experimental procedures of flow cytometric analysis, e.g. cell-harvesting step, may slightly affect neu- ronal survival.

Next, we examined the distribution of cathepsin D between cytosolic and microsomal fractions after oxidative stress and proteasome inhibition. First, the separation of each fractionation was confirmed by immunoblotting of GAPDH, a cytosol marker. As shown in Fig. 1d, immunopositive bands of GAPDH were only detected in the cytosolic fraction, not in the microsomal fraction. By cathepsin D-immunoblotting, major bands of 40 to $50 \mathrm{kDa}$ molecular weight were observed. As reported previously for rat cathepsin $\mathrm{D},{ }^{12,23)}$ the $43 \mathrm{kDa}$ band represented a single-chain form and that of $32 \mathrm{kDa}$ represented a matured double-chain form of the enzyme. Under the present conditions, only the single-chain form was detected in primary cultured neurons. Most cathep$\sin \mathrm{D}$ was detected in the microsomal fraction of control cells (group 1,3), while in cells exposed to oxidative stress (group 2) or proteasome inhibition (group 4), cathepsin D in the cytosolic fraction was strongly detected, indicating that the distribution of cathepsin D changed from lysosomes to the cytosol. This is consistent with the results shown in Figs. 1a and b. These results indicate that in primary cultured neurons, oxidative stress and proteasome inhibition induced apoptosis, and concomitantly, cathepsin D was translocated from lysosomes to the cytosol.

Effects of Oxidative Stress and Proteasome Inhibition on Astrocytes We then examined the effects of oxidative stress and proteasome inhibition on the translocation of cathepsin D in cultured astrocytes. Figure 2a shows the effects of oxidative stress or proteasome inhibition on chromatin condensation, and the distribution of cathepsin D and GFAP in primary cultured astrocytes. When astrocytes were treated with $50 \mu \mathrm{M}$ tBHP for $1 \mathrm{~h}$, chromatin condensation was observed on the Hoechst 33342 staining panel, indicating apoptotic cells. Translocation of cathepsin D from lysosomelike granules to the cytosol was observed in tBHP-treated cells compared to the control $(-)$. The addition of lactacystin at $100 \mu \mathrm{M}$ also caused chromatin condensation and translocation of cathepsin D to the cytosol. These results suggest that apoptosis and the translocation of cathepsin D to the cytosol were also induced by oxidative stress and the inhibition of proteasome in cultured astrocytes, similarly to neurons; however, the concentration of tBHP and lactacystin, which was required to induce apoptosis and the translocation of cathep$\sin \mathrm{D}$ in astrocytes, was much higher than that of neurons.

We also performed flow cytometric analysis by annexin VPI staining in astrocytes, as shown in Fig. 2b. The addition of tBHP at $150 \mu \mathrm{M}$ or lactacystin at $100 \mu \mathrm{M}$ increased B4 cells and decreased B3 cells, indicating the induction of apoptosis. This is consistent with the results shown in Fig. 2a.

Figure 2c demonstrates the subcellular localization of cathepsin D in astrocytes after oxidative stress or proteasome inhibition. Similar to neurons, a single-chain form of $43 \mathrm{kDa}$ was mainly observed (Fig. 1d). Almost cathepsin D existed in the microsomal fraction of control cells (group 1), while in cells loaded with oxidative stress (group 2, 3), more cathep$\sin \mathrm{D}$ was detected in the cytosolic fraction depending on the concentration of tBHP. In the cytosolic fraction of group 3, since surviving cells were decreased by $150 \mu \mathrm{M}$ tBHP exposure, cell recovery was lower than in other samples, resulting in undetectable GAPDH. On the other hand, cathepsin D was not increased in the cytosolic fraction of $100 \mu \mathrm{M}$ lactacystin- 
(a)
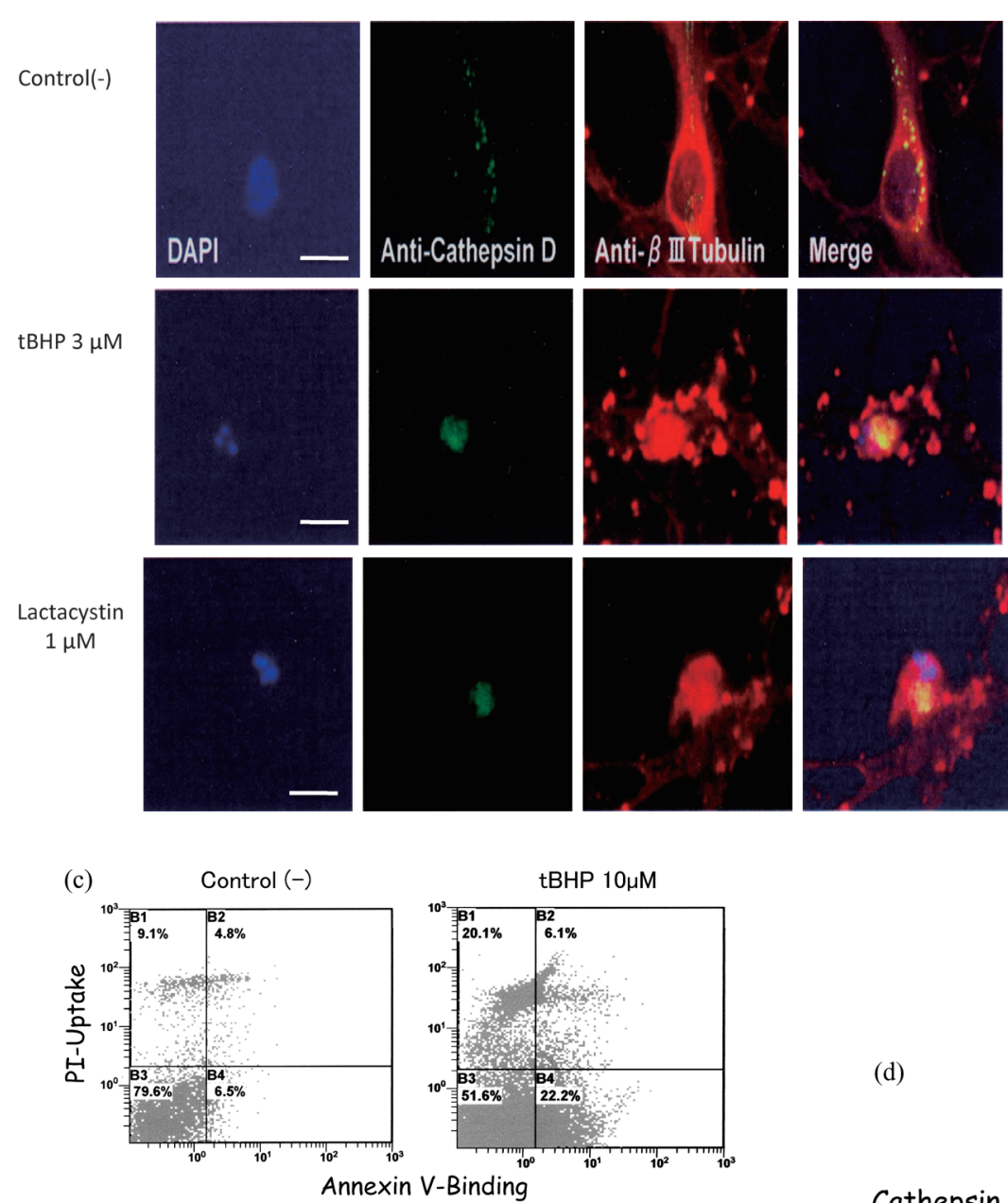

Control (+)

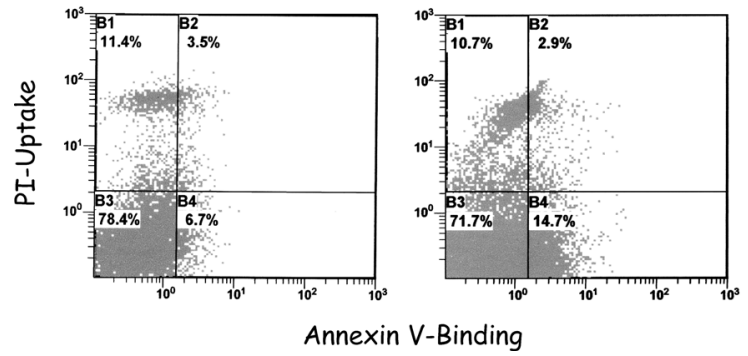

(b)
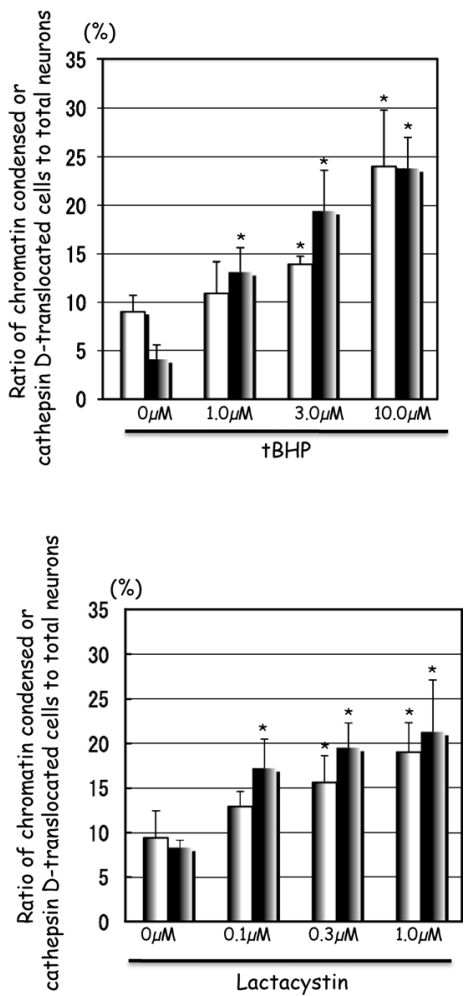

(d)

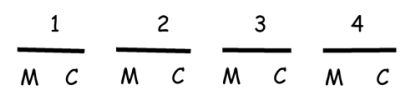

Cathepsin D

GAPDH

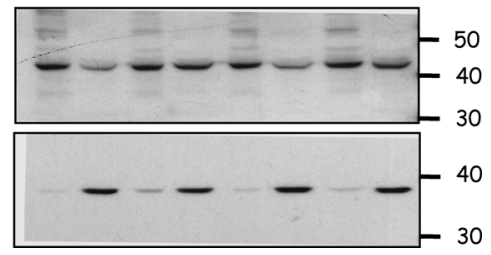

Fig. 1. (a) DAPI Staining, and Immunofluorescence Detection of Cathepsin D and TuJ-1 in Primary Cultured Neurons

Cells were exposed to $3 \mu \mathrm{m}$ tBHP for $1 \mathrm{~h}$ or to $1 \mu \mathrm{m}$ lactacystin for $24 \mathrm{~h}$. Cells were fixed in methanol after incubation with $2 \mu \mathrm{g} / \mathrm{ml}$ DAPI at $37^{\circ} \mathrm{C}$ for $15 \mathrm{~min}$ and then subjected to dual immunocytochemical staining with anti-cathepsin D and anti-TuJ-1 antibodies. Details of immunocytochemical staining are described in Materials and Methods. Original magnification, $\times 400$. Bar in DAPI panels, $10 \mu \mathrm{m}$

(b) Ratio of Chromatin Condensed or Cathepsin D-Translocated Cells to Total Neurons

Open and closed bars represent chromatin condensation and cathepsin D translocation, respectively. Data were calculated after counting over 300 cells in each cell group. $* p<0.05 v s .0 \mu \mathrm{m}$ for each cell group.

(c) Representative Cytogram of Neurons

Representative cytograms of annexin V (abscissa) vs. PI (ordinate) fluorescence determined. Cells were exposed to $10 \mu \mathrm{M}$ tBHP or $1 \mu \mathrm{M}$ lactacystin as described in Materials and Methods, and stained with both FITC-conjugated annexin V and PI. Quadrants designated B1-B4; lower left (B3), a group of annexin V- and PI-negative cells, indicating living cells; upper left (B1), PI-positive, indicating a group of necrotic cells; lower right (B4), annexin V-positive, indicating a group of early apoptotic cells; upper right (B2), both annexin V- and PI-positive, indicating a group of late apoptotic cells.

(d) Distribution of Cathepsin D and GAPDH in tBHP- or Lactacystin-Exposed Neurons

Primary cultured neurons were exposed to tBHP- or lactacystin as described in Materials and Methods. Collected cells were homogenized with SET buffer (10 mM Tris-HCl pH $7.4,1 \mathrm{~mm}$ EDTA-2Na and $250 \mathrm{~mm}$ sucrose) and centrifuged at $600 \times \boldsymbol{g}$ for $10 \mathrm{~min}$ at $4{ }^{\circ} \mathrm{C}$. After discarding the pellet, the supernatant was centrifuged at $100000 \times \boldsymbol{g}$ for $1 \mathrm{~h}$ at $4{ }^{\circ} \mathrm{C}$, and the cytosol and microsomal fraction were obtained from the supernatant and pellet fraction, respectively. After subcellular fractionation, mirosomal (M) and cytosolic (C) fractions were analyzed by Western blotting with anti-cathepsin D and anti-GAPDH antibody. 1: Control (-), 2: tBHP 50 $\mu \mathrm{M}, 3$ : Control (+), 4: Lactacystin 5 $\mu \mathrm{M}$. 
(a)
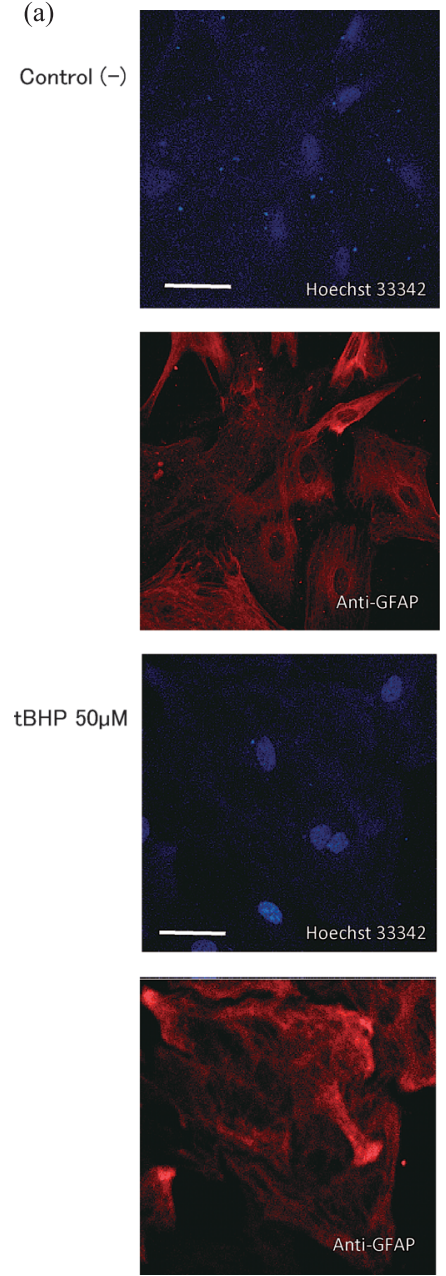

(b)

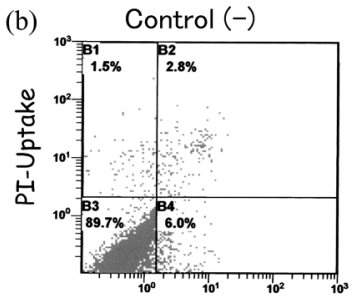

Annexin V-Binding

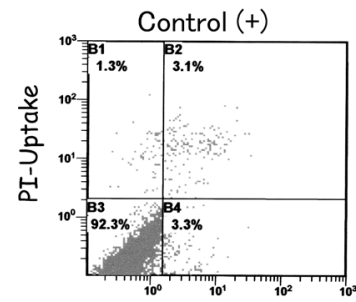

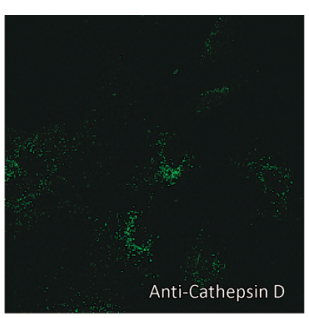
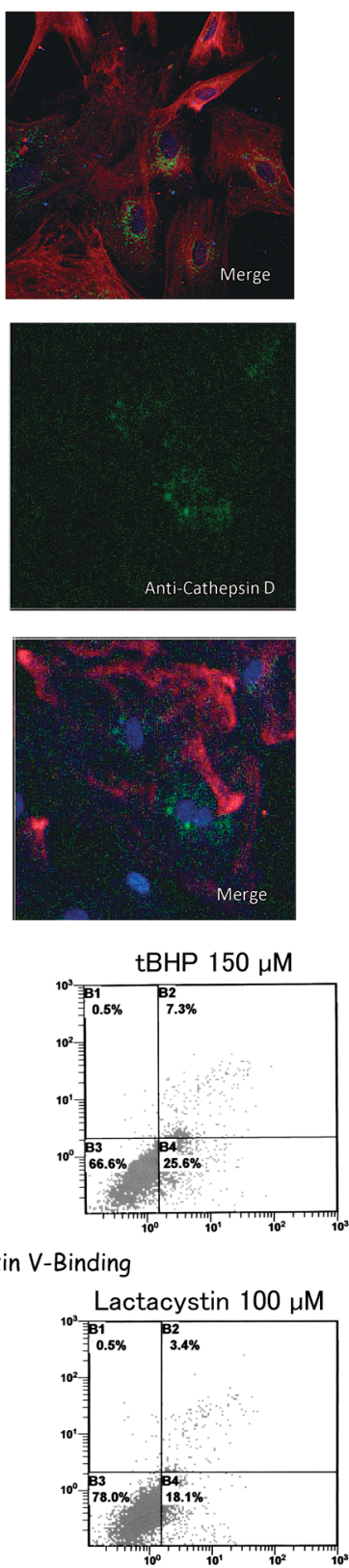
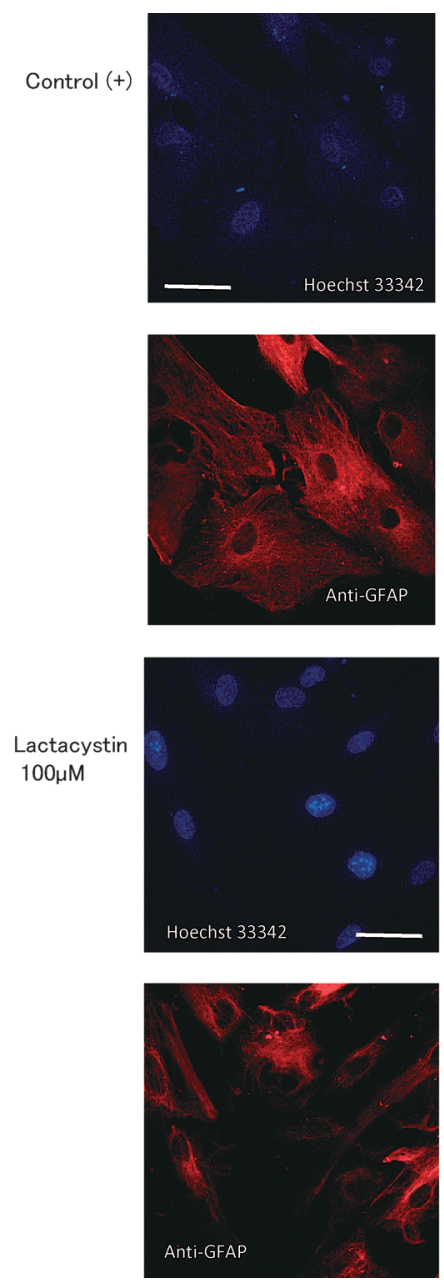

(c)

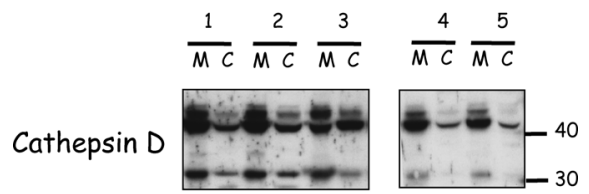

GAPDH
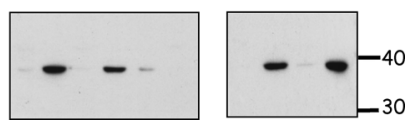

Fig. 2. (a) Hoechst 33342 Staining, and Immunofluorescence Detection of Cathepsin D and GFAP in Primary Cultured Astrocytes

Cells were exposed to $50 \mu \mathrm{m}$ tBHP for $1 \mathrm{~h}$ or to $100 \mu \mathrm{m}$ lactacystin for $24 \mathrm{~h}$ and then subjected to dual immunocytochemical staining with anti-cathepsin D and anti-GFAP antibodies after Hoechst 33342 staining. Experimental details are described in Fig. 1a. Original magnification, $\times 400$. Bar in Hoechst 33342 panels, $50 \mu \mathrm{m}$.

(b) Representative Cytogram of Astrocytes

Representative cytograms of annexinV (abscissa) $v s$. PI (ordinate) fluorescence determined. Cells were exposed to $150 \mu \mathrm{M}$ tBHP or $100 \mu \mathrm{M}$ lactacystin as described in Materials and Methods, and stained with both FITC-conjugated annexin V and PI. Quadrants designated B1-B4 are described in figure legends of Fig. 1c.

(c) Distribution of Cathepsin D and GAPDH in tBHP- or Lactacystin-Exposed Astrocytes

Cells were exposed to tBHP at 50 or $150 \mu \mathrm{m}$ or lactacystin at $100 \mu \mathrm{M}$. Collected cells were homogenized with SET buffer and fractionated by centrifugations, subsequently analyzed by Western blotting with anti-cathepsin D and anti-GAPDH antibodies as described in figure legends of Fig. 1d. 1: Control (-), 2: tBHP 50 $\mu \mathrm{M}, 3$ : tBHP 150 $\mu \mathrm{M}, 4$ : Control (+), 5: Lactacystin, $100 \mu \mathrm{M}$. 
treated cells (group 5). Since Fig. 2a showed that cathepsin D was translocated to cytosol by the treatment of $100 \mu \mathrm{M}$ lactacystin, these results are contradictory. It seems that the damaged astrocytes, of which cathepsin D was translocated to the cytosol, probably failed to be recovered under our experimental procedures of subcellular fractionation.

\section{DISCUSSION}

We have demonstrated that oxidative stress and proteasome inhibition induced apoptosis in primary cultured neurons and astrocytes. The fact that oxidative stress induced apoptosis in neurons and astrocytes is consistent with other studies. ${ }^{24,25)}$ On the other hand, lactacystin is reported to elicit a biphasic effect, both neuroprotective and pro-apoptotic, on neuronal cells. ${ }^{26,27)}$ Butts et al. demonstrated that acute exposure to proteasome inhibitors (6-h exposure) caused a pro-survival response, while a chronic exposure (16h) triggered apoptosis. ${ }^{26)}$ Our results suggest that exposure to lactacystin for $24 \mathrm{~h}$ has pro-apoptotic effects on primary cultured neurons.

We found the translocation of cathepsin D caused by oxidative stress or proteasome inhibition in primary cultured neurons and astrocytes. Based on the results, two possible mechanisms of cathepsin D accumulation in the cytosol should be considered; increased destabilization of the lysosomal membrane, followed by the diffusion of cathepsin D from lysosome, and the decreased capacity of protein degradation systems in the cytosol, leading to the accumulation of cytosolic cathepsin D.

The exposure to tBHP induced the translocation of cathep$\sin \mathrm{D}$ to cytosol in cultured neurons and astrocytes. These results suggested that oxidative stress was a factor inducing cathepsin D accumulation in the cytosol during aging. Oxidative stress triggered lysosomal destabilization and the diffusion of cathepsin D to cytosol in other cells. ${ }^{19-22)}$ Although the mechanisms of lysosomal destabilization are unclear, one possible mechanism is oxidative stress-induced peroxidation of the lysosomal membrane. A considerable amount of iron is held inside the lysosomal compartment as a result of the degradation of metalloproteins such as cytochromes. ${ }^{28)}$ Under oxidative conditions, Fenton reactions catalyzed by iron yield ROS such as $\cdot \mathrm{OH}$, which would lead to lipid peroxidation and the destabilization of lysosomal membranes. Furthermore, the acidic $\mathrm{pH}$ of lysosomes amplifies ironmediated lipid peroxidation. ${ }^{29)}$

We demonstrated that the translocation of cathepsin D to cytosol was caused by the inhibition of proteasomes in cultured neurons and astrocytes, suggesting that reduced proteasome activity is another factor in cathepsin D accumulation in the cytosol. It is known that proteasome activity declines with the aging process. ${ }^{30)}$ The proteasome protein degradation system contributes to eliminate misfolded/unassembled glycoproteins such as cathepsin D, which are dislocated to the cytosol. This system consists of two steps, de-glycosylation by peptide $N$-glycanase (PNGase) and then degradation by proteasomes following ubiquitination ${ }^{3)}$; therefore, in addition to the decrease of proteasome activity, the suppression of PNGase and/or ubiquitination with the aging process may also be responsible for the accumulation of cathepsin D in the cytosol. Regarding ubiquitination, Scrofano et al. re- ported that aging was associated with increased levels of ubiquitin conjugates in the liver of Emory mice. ${ }^{31)}$ They assumed that the age-related increase in the level of ubiquitin conjugates was primarily due to increased ubiquitin-activating (E1) and ubiquitin-conjugating (E2) activities. These results suggest that ubiquitination may not decrease with aging. On the other hand, the age-related change of PNGase activity is not known.

Does cytosolic cathepsin D play an important role in apoptosis? The involvement of cathepsin D in apoptosis has been reported in various cases..$^{25,32-37)}$ One possible pathway of cathepsin D-mediated apoptosis is that the cleavage of bid, a pro-apoptotic factor of the Bcl-2 family, then triggers Bax activation and translocation onto the mitochondrial membrane followed by apoptosis ${ }^{38-40)}$; however, it was reported that cathepsin D exhibited little or no activity at $\mathrm{pH} 5$ and above, such as in the cytosol, ${ }^{41)}$ suggesting that cathepsin D did not work as an active enzyme to degrade cytosolic proteins. Therefore, it is still controversial that enzymatic activity of cytosolic cathepsin D is involved in the apoptosis process.

It is reported that astrocytes are more resistant to oxidative stress than neurons. ${ }^{42,43)}$ The different sensitivity of astrocytes and neurons to oxidative stress was due to the difference in intracellular glutathione content. Furthermore, the tolerance of astrocytes to clasto-lactacystin, an active metabolite of lactacystin, has been reported. ${ }^{44)}$ Our results supported these previous studies. If the brain is suffering from oxidative stress, neurons should be more severely damaged than astrocytes. Furthermore, mature functional neurons are post-mitotic cells while astrocytes are mitotic cells. Taken together, the deterioration of brain function by oxidative stress may be due to the loss of neurons rather than astrocytes, and it may occur during aging.

In the present study, we demonstrated that both oxidative stress and proteasome inhibition induced cathepsin D translocation from lysosomes to the cytosol in primary cultured neurons and astrocytes. This suggests that oxidative stress and the suppression of proteasome activity are responsible for the age-related accumulation of cathepsin D in the cytosol. ${ }^{8)}$ Although it is unknown whether cathepsin D accumulated in the cytosol mediates neural or astocytic cell death directly, cytosolic cathepsin D would be a biomarker of agerelated damage in the brain.

Acknowledgements The remaining coauthors wish to dedicate this manuscript to the life and career of Yuji Sato, who died on June 21, 2006. This study was partly supported by a Grant-in-Aid for Scientific Research (C) (No. 20590082 to Y.M.) and (B) (No. 20390031 to T.E.) from the Japan Society for the Promotion of Science.

\section{REFERENCES AND NOTES}

1) Present address: Advanced Medical Science Research Center, Mitsubishi Chemical Medience Corporation; Shimura, Itabashi-ku, Tokyo 174-8555, Japan.

2) Apweiler R., Hermjakob H., Sharon N., Biochim. Biophys. Acta, 1473, 4-8 (1999).

3) Funakoshi Y., Suzuki T., Biochim. Biophys. Acta, 1790, 81-94 (2009).

4) Pedemonte C. H., Sachs G., Kaplan J. H., Proc. Natl. Acad. Sci. U.S.A., 87, 9789-9793 (1990). 
5) Miyagi T., Konno K., Emori Y., Kawasaki H., Suzuki K., Yasui A., Tsuik S., J. Biol. Chem., 268, 26435-26440 (1993).

6) Dimri G. P., Lee X., Basile G., Acosta M., Scott G., Roskelley C., Medrano E. E., Linskens M., Rubelj I., Pereira-Smith O., Proc. Natl. Acad. Sci. U.S.A., 92, 9363-9367 (1995).

7) Sato Y., Naito Y., Grundke-Iqbal I., Iqbal K., Endo T., FEBS Lett., 496, 152-160 (2001).

8) Sato Y., Suzuki Y., Ito E., Shimazaki S., Ishida M., Yamamoto T., Yamamoto H., Toda T., Suzuki M., Suzuki A., Endo T., Mech. Ageing Dev., 127, 771-778 (2006).

9) Kenessey A., Banay-Schwartz M., DeGuzman T., Lajtha A., J. Neurosci. Res., 23, 454-456 (1989).

10) Nakamura Y., Takeda M., Suzuki H., Morita H., Tada K., Hariguchi S., Nishimura T., Mech. Ageing Dev., 50, 215-225 (1989).

11) Nakanishi H., Tominaga K., Amano T., Hirotsu I., Inoue T., Yamamoto K., Exp. Neurol., 126, 119 -128 (1994).

12) Adamec E., Mohan P. S., Cataldo A. M., Vonsattel J. P., Nixon R. A., Neuroscience, 100, 663-675 (2000).

13) Nakanishi H., Amano T., Sastradipura D. F., Yoshimine Y., Tsukuba T., Tanabe K., Hirotsu I., Ohono T., Yamamoto K., J. Neurochem., 68, $739-749$ (1997).

14) Gopalan P., Dufresne M. J., Warner A. H., Can. J. Physiol. Pharmacol., 65, 124-129 (1987).

15) Harman D., Ann. N.Y. Acad. Sci., 1067, 10-21 (2006).

16) Miura Y., J. Radiat. Res. (Tokyo), 45, 357-372 (2004).

17) Miura Y., Abe K., Urano S., Furuse T., Noda Y., Tatsumi K., Suzuki S., Int. J. Radiat. Biol., 78, 913-921 (2002).

18) Miura Y., Kano M., Abe K., Urano S., Suzuki S., Toda T., Electrophoresis, 26, 2786-2796 (2005).

19) Choi J. H., Kim D. H., Yun I. J., Chang J. H., Chun B. G., Choi S. H., Eur. J. Pharmacol., 571, 106-115 (2007).

20) Roberg K., Johansson U., Ollinger K., Free Radic. Biol. Med., 27, 1228-1237 (1999).

21) Roberg K., Ollinger K., Am. J. Pathol., 152, 1151-1156 (1998).

22) Vene R., Arena G., Poggi A., D’Arrigo C., Mormino M., Noonan D. M., Albini A., Tosetti F., Mol. Cancer Ther, 6, 286-298 (2007).

23) Fujita H., Tanaka Y., Noguchi Y., Kono A., Himeno M., Kato K., Biochem. Biophys. Res. Commun., 179, 190-196 (1991).

24) Ran Q., Gu M., Van Remmen H., Strong R., Roberts J. L., Richardson A., J. Neurosci. Res., 84, 202-208 (2006).

25) Takuma K., Kiriu M., Mori K., Lee E., Enomoto R., Baba A., Matsuda T., Neurochem. Int., 42, 153-159 (2003).

26) Butts B. D., Hudson H. R., Linseman D. A., Le S. S., Ryan K. R., Bouchard R. J., Heidenreich K. A., Mol. Cell. Neurosci., 30, 279-289 (2005).
27) Yew E. H., Cheung N. S., Choy M. S., Qi R. Z., Lee A. Y., Peng Z. F., Melendez A. J., Manikandan J., Koay E. S., Chiu L. L., Ng W. L., Whiteman M., Kandiah J., Halliwell B., J. Neurochem., 94, 943-956 (2005).

28) Petrat F., de Groot H., Rauen U., Biochem. J., 356, 61-69 (2001).

29) Schafer F. Q., Buettner G. R., Free Radic. Biol. Med., 28, 1175-1181 (2000).

30) Ponnappan S., Ovaa H., Ponnappan U., Int. J. Biochem. Cell Biol., 39, 799-809 (2007).

31) Scrofano M. M., Shang F., Nowell T. R. Jr., Gong X., Smith D. E., Kelliher M., Dunning J., Mura C. V., Taylor A., Mech. Ageing Dev., 101, 277-296 (1998).

32) Choi S. H., Choi D. H., Lee J. J., Park M. S., Chun B. G., Free Radic. Biol. Med., 32, 394- 405 (2002).

33) Isahara K., Ohsawa Y., Kanamori S., Shibata M., Waguri S., Sato N., Gotow T., Watanabe T., Momoi T., Urase K., Kominami E., Uchiyama Y., Neuroscience, 91, 233-249 (1999).

34) Kagedal K., Johansson U., Ollinger K., FASEB J., 15, 1592-1594 (2001).

35) Kanthasamy A., Anantharam V., Ali S. F., Kanthasamy A. G., Ann. N.Y. Acad. Sci., 1074, $234-244$ (2006).

36) Heinrich M., Neumeyer J., Jakob M., Hallas C., Tchikov V., WinotoMorbach S., Wickel M., Schneider-Brachert W., Trauzold A., Hethke A., Schutze S., Cell Death Differ., 11, 550 -563 (2004).

37) Berndtsson M., Beaujouin M., Rickardson L., Havelka A. M., Larsson R., Westman J., Liaudet-Coopman E., Linder S., Int. J. Cancer, 124, 1463-1469 (2009).

38) Bidere N., Lorenzo H. K., Carmona S., Laforge M., Harper F., Dumont C., Senik A., J. Biol. Chem., 278, 31401-31411 (2003).

39) Castino R., Bellio N., Nicotra G., Follo C., Trincheri N. F., Isidoro C., Free Radic. Biol. Med., 42, 1305-1316 (2007).

40) Stoka V., Turk B., Schendel S. L., Kim T. H., Cirman T., Snipas S. J., Ellerby L. M., Bredesen D., Freeze H., Abrahamson M., Bromme D., Krajewski S., Reed J. C., Yin X. M., Turk V., Salvesen G. S., J. Biol. Chem., 276, 3149-3157 (2001).

41) Conner G. E., "Handbook of Proteolytic Enzymes," ed. by Barrett A. J., Rawlings N. D., Woessner J. F., 2nd ed., Elsevier Academic Press, Amsterdam, 2004, pp. 43-52.

42) Bolanos J. P., Heales S. J., Land J. M., Clark J. B., J. Neurochem., 64, 1965-1972 (1995).

43) Hollensworth S. B., Shen C., Sim J. E., Spitz D. R., Wilson G. L., LeDoux S. P., Free Radic. Biol. Med., 28, 1161-1174 (2000).

44) Snider B. J., Tee L. Y., Canzoniero L. M., Babcock D. J., Choi D. W., Eur. J. Neurosci., 15, 419-428 (2002). 